\title{
Thoracic Endometriosis
}

National Cancer Institute

\section{Source}

National Cancer Institute. Thoracic Endometriosis. NCI Thesaurus. Code C158138.

A condition characterized by the presence of functioning endometrial tissue in the lung, pleura, chest wall, and/or diaphragm. 